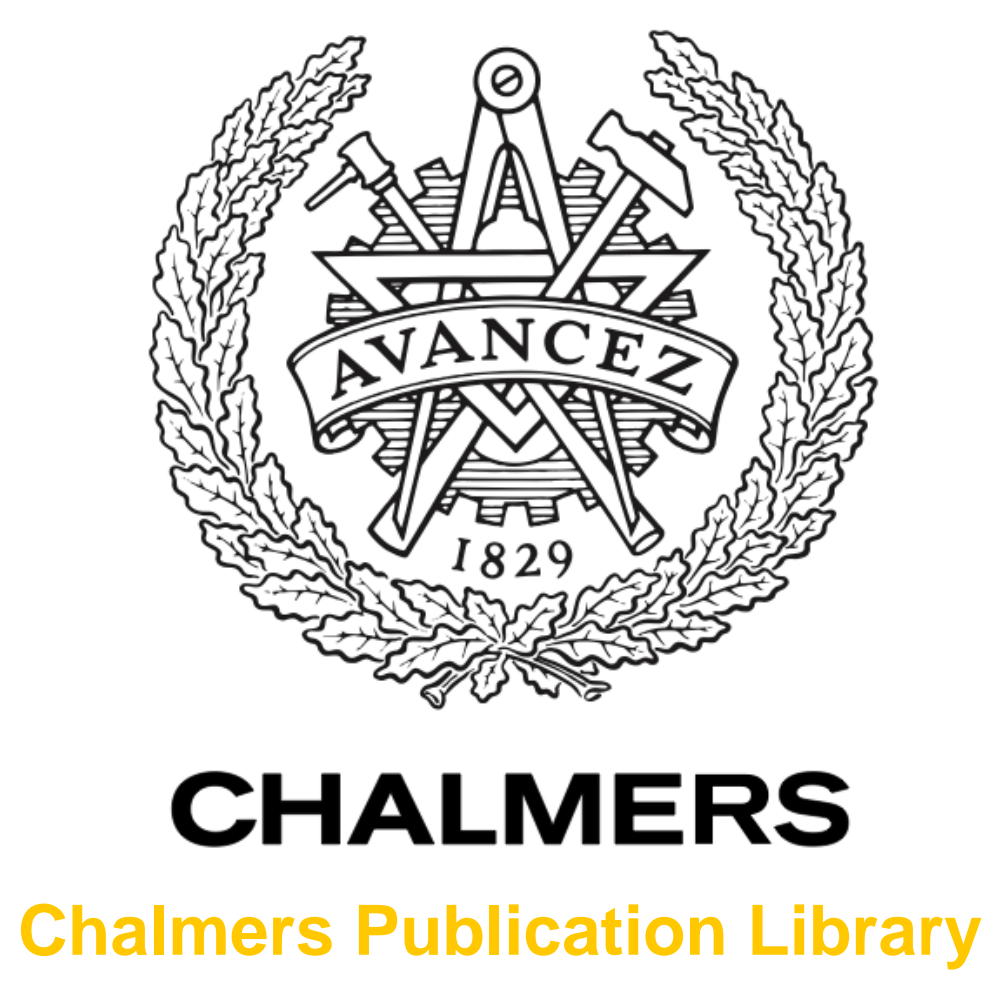

\author{
Wearable textile antennas' efficiency characterization using a reverberation \\ chamber
}

This document has been downloaded from Chalmers Publication Library $(\mathrm{CPL})$. It is the author's version of a work that was accepted for publication in:

2011 IEEE International Symposium on Antennas and Propagation, Spokane, USA, 3-8 July, 2011 (ISSN: 1522-3965)

Citation for the published paper:

Soh, P. ; Vandenbosch, G. ; Chen, X. (2011) "Wearable textile antennas' efficiency characterization using a reverberation chamber". 2011 IEEE International Symposium on Antennas and Propagation, Spokane, USA, 3-8 July, 2011 pp. 810-813.

http://dx.doi.org/10.1109/APS.2011.5996397

Downloaded from: http://publications.lib.chalmers.se/publication/143611

Notice: Changes introduced as a result of publishing processes such as copy-editing and formatting may not be reflected in this document. For a definitive version of this work, please refer to the published source. Please note that access to the published version might require a subscription.

Chalmers Publication Library (CPL) offers the possibility of retrieving research publications produced at Chalmers University of Technology. It covers all types of publications: articles, dissertations, licentiate theses, masters theses, conference papers, reports etc. Since 2006 it is the official tool for Chalmers official publication statistics. To ensure that Chalmers research results are disseminated as widely as possible, an Open Access Policy has been adopted.

The CPL service is administrated and maintained by Chalmers Library. 


\title{
Wearable Textile Antennas' Efficiency Characterization using a Reverberation Chamber
}

\author{
P.J Soh ${ }^{1,2}$, G.A.E Vandenbosch ${ }^{1}$, X.Chen ${ }^{3}$, P.S Kildal ${ }^{3}$, S.L Ooi ${ }^{2,4}$, H.Aliakbarian ${ }^{1}$ \\ ${ }^{1}$ ESAT-TELEMIC, Katholieke Universiteit Leuven, 3001 Leuven, Belgium \\ Email: [pingjack.soh, guy.vandenbosch]@esat.kuleuven.be \\ ${ }^{2}$ School of Computer and Communication, Universiti Malaysia Perlis (UniMAP), 02000 Perlis, Malaysia \\ Email: pjsoh@unimap.edu.my, sooliam@gmail.com \\ ${ }^{3}$ Antenna Group, Department of Signal and Systems, Chalmers University of Technology, S-41296 Gothenburg, Sweden \\ Email: [xiaoming.chen, per-simon.kildal]@chalmers.se \\ ${ }^{4}$ Sulucon Research, 10760 NW $12^{\text {th }}$ Drive, Plantation, FL 33322, USA \\ Email: sooliam@sulucon.com
}

\begin{abstract}
Characterization of efficiency is an important consideration to ensure the proper operation of an all-textile wearable antenna. In this work, a small, novel textile antenna is designed based on a planar inverted-F structure (PIFA). The structure incorporates an edge-feeding plate, symmetrical design, and well-placed slot, in order to ease fabrication. A thin conductive textile, Pure Copper Polyester Taffeta Fabric (PCPTF), and fleece is used to fabricate the structure. The efficiency is then verified and measured using a commercial reverberation chamber. Observed results indicate excellent agreements between simulations and measurements.
\end{abstract}

Keywords-component; Planar Inverted-F Antenna (PIFA), textile antenna, broadband antennas, wearable antennas

\section{INTRODUCTION}

The emergence of wearable communication systems has boosted the interest in wearable antennas. Various applications in military, medical, emergency response, and consumer electronics applications have been seen to embed conventional cables, miniaturized electronic components, and connectors altogether in a wearable smart clothing system. The smart clothing electronic system should incorporate wireless communication components made of flexible textile rather than bulky boxes made from rigid materials. This has triggered the need for a flexible and wearable radiating system to suit this purpose, enabling a transparent or 'non-existent' system to the users.

Degradation of the antenna performance when worn on the human body has been one of the major deterrents in a successful implementation, be it in terms of frequency detuning, bandwidth reduction, and efficiency degradation or radiation distortion. In other words, ideally, a wearable antenna must be designed to be immune enough for on-body operation. This makes researchers to be address the fundamental issue of efficiency. Various researchers have come up with a multitude

This work is carried out within the framework of European COST action ic0603 (COST-ASSIST), supported by the Malaysian Ministry of Higher Education (MOHE) and Universiti Malaysia Perlis (UniMAP). of strategies in order to minimize the effect of this degradation. One strategy is to design an antenna broad enough to cater for the antenna degradation in the proximity of the users' body [1].

A reverberation chamber $(R C)$ is basically a metal cavity with many excited modes which are stirred to create a statistically isotropic field environment [2]. Initially used for electromagnetic compatibility $(E M C)$, its capability is now expanded in order to measure radiation efficiency, diversity gain, capacity of multiple-input-multiple-output (MIMO) systems, etc. The measurements of various conventional antennas and active devices have been investigated in previous works [3]. Although it has been shown recently that efficiencies of small antennas can be accurately measured using $\mathrm{RCs}$, to date there is very little experience with measuring alltextile antenna in such facility. Still, some previous measurements are mentioned in [4].

Antenna efficiency and total radiated power are obtained by measuring and averaging the power transfer function $\left|S_{21}\right|^{2}$ between two or more antennas for many stirring positions [5]. The variation of these average power transfer functions is a metric of the measurement accuracy. The Bluetest $\mathrm{AB}$ chamber integrates various stirring methods - mechanical stirring (including platform stirring) and polarization stirring[6]. Through these stirrings, sufficient independent and uncorrelated samples are introduced, ensuring measurement accuracy. In addition to that, this work investigates frequency stirring for textile antenna measurements. This technique is executed by averaging the chamber's power transfer function over a certain frequency stirring bandwidth $(\delta f)$ to improve measurement accuracy. However, careful selection of $\delta f$ must be taken into account, as the resulting frequency resolution can be reduced. 


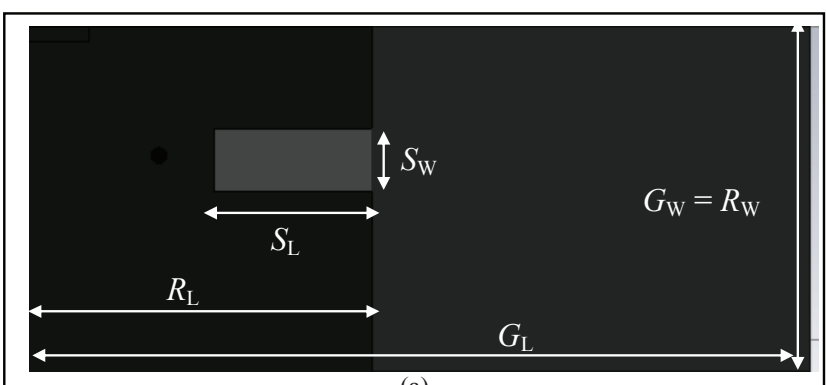

(a)

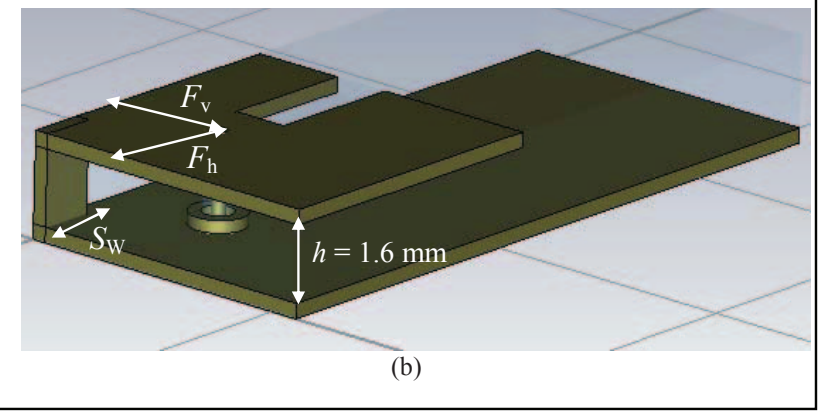

Figure 1. Dimension of the proposed SPIFA in CST (a) Top view (b) Perspective view

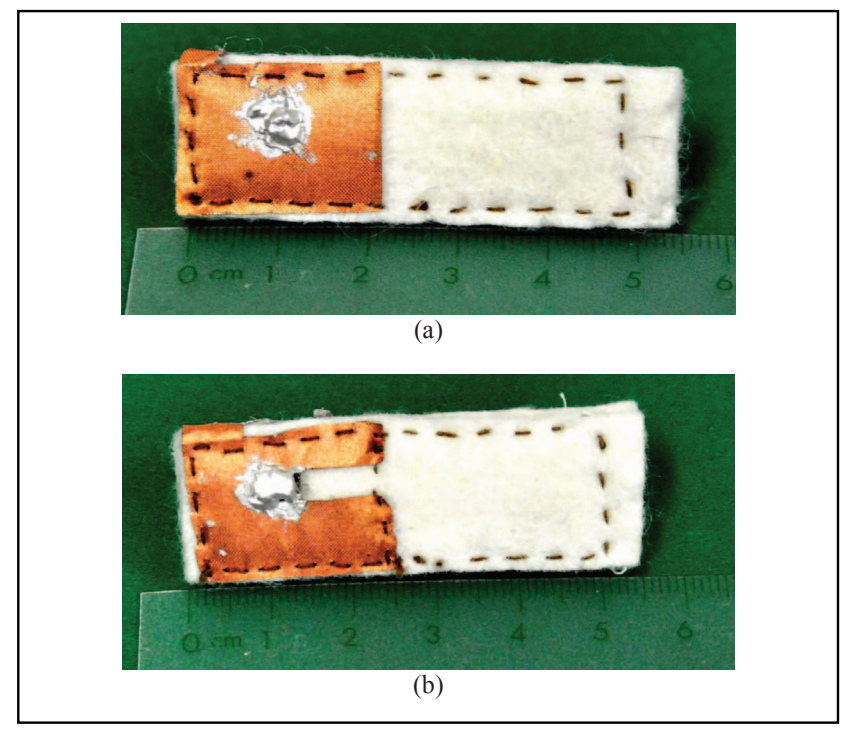

Figure 2. The fabricated PIFAs (a) Plain (PPIFA) and (b) Slotted (SPIFA)

\section{TeXtile MAterial AND ANTENNA Design}

The conductive textile material used in this work, PCPTF, is a plain woven polyester textile, plated using copper. This textile material from LessEMF Inc USA, is $0.08 \mathrm{~mm}$ thick, and possesses a surface resistance, $R_{\mathrm{s}}$, of less than $0.05 \Omega /$ sq. It has been used to form the conductive components of the PIFA, i.e the radiator, ground plane and shorting wall, all in a single and continuous structure. A $50 \Omega$ SMA connector is used to feed power into the radiator. It is attached to the textile radiator and ground plane using EE129-4 conductive epoxy from Epotek Inc USA. To ease integration into normal clothing, the radiator and ground plane area are spaced by a $6 \mathrm{~mm}$ thick fleece, which is estimated to possess a relative dielectric constant, $\varepsilon_{\mathrm{r}}$ of 1.26 [7]. Initial calculation of the dimensions for a center frequency of $2.45 \mathrm{GHz}$ is carried out using the procedure defined in [8]. The structure is then simulated and optimized using CST Microwave Studio. The topology is shown in Fig. 1.

Calculation yields a ground plane dimension of $G_{\mathrm{L}} \times G_{\mathrm{W}}=$ $50 \times 24 \mathrm{~mm}$, a radiator sized at $R_{\mathrm{L}} \times R_{\mathrm{W}}=36 \times 24 \mathrm{~mm}$, a shorting wall width $\left(S_{\mathrm{W}}\right)$ of $5 \mathrm{~mm}$ and a feeding location at the center of the radiator, $F_{\mathrm{h}}$ and $F_{\mathrm{v}}$ are 10 and $12.5 \mathrm{~mm}$, respectively. Optimization further miniaturized the overall dimensions, resulting in $G_{\mathrm{L}}=50 \mathrm{~mm}, R_{\mathrm{L}}=23 \mathrm{~mm}, G_{\mathrm{W}}=R_{\mathrm{W}}=$ $19 \mathrm{~mm}, S_{\mathrm{W}}=5 \mathrm{~mm}, F_{\mathrm{h}}=10.5 \mathrm{~mm}$, and $F_{\mathrm{v}}=7.7 \mathrm{~mm}$.

Two types of radiator topologies are fabricated and tested, one with a plain radiator $(P P I F A)$, and another with a slot (SPIFA) dimensioned at $S_{\mathrm{L}} \times S_{\mathrm{W}}=9 \mathrm{~mm} \times 6 \mathrm{~mm}$, placed at the edge of the radiator. Fabrication of the antennas is performed using simple, manual dimensioning and cutting tools. Thus, fabrication inaccuracy is expected to be slightly larger in comparison to fabrication of conventional antennas using Printed Circuit Board $(P C B)$ technology. For each topology, two samples (labeled as PPIFA1, PPIFA2, SPIFA1 and $S P I F A 2$, respectively) are fabricated as carefully as possible, and then tested for performance. This is done to investigate the extent of the anticipated fabrication inaccuracies. In addition, prototypes 1 and 2 are fabricated using different sizes of fleece, to investigate whether effect of the overall size of the antenna will be significant. The $P 1$ and $P 2$ fleeces are sized at $25 \mathrm{~mm} \mathrm{x}$ $80 \mathrm{~mm}$, and $25 \mathrm{~mm} \times 55 \mathrm{~mm}$, respectively. Besides, $P 1$ and $P 2$ prototypes are also fabricated one month apart to investigate the effect of textile/epoxy aging. The fabricated antennas are shown in Fig. 2.

\section{EFFICIENCY MEASUREMENT SETUP}

The reverberation chamber from Bluetest $A B$ used in the measurement is sized at $1.8 \times 1.7 \times 1.2 \mathrm{~m}^{3}$. Mechanical stirring is performed by two plates (one horizontal and another vertical) moving step-wise, while the Antenna under Test (AUT) is placed onto a rotating platform. In such way, the rotating platform creates a 20-position measurement, spaced by $18^{\circ}$. On the other hand, both stirrer plates were moved at 10-positions both horizontally and vertically. Polarization stirring is also introduced by the implementation of three wall antennas mounted at three orthogonal walls. Due to the antennas' broad bandwidth, measurements of the AUT are carried out between $1.5-3.0 \mathrm{GHz}$, with a $1 \mathrm{MHz}$ interval. Since the numbers of points are limited to 1601 points per measurement, two consecutive measurements are carried out for the same antenna, one from 1.5 to $2 \mathrm{GHz}$, and another from 2 to $3.5 \mathrm{GHz}$. However, minimal error is expected since no physical changes are applied to the antenna in between measurements.

During the measurements, the chamber was loaded with a brain equivalent liquid-filled head phantom. Initially, a 700 $\mathrm{MHz}$ to $6 \mathrm{GHz}$ discone antenna with known efficiency is chosen as the reference antenna, and measured for its power transfer function. Next, the $A U T$ is placed in the chamber and measured using the same procedure and load. The ratio of 


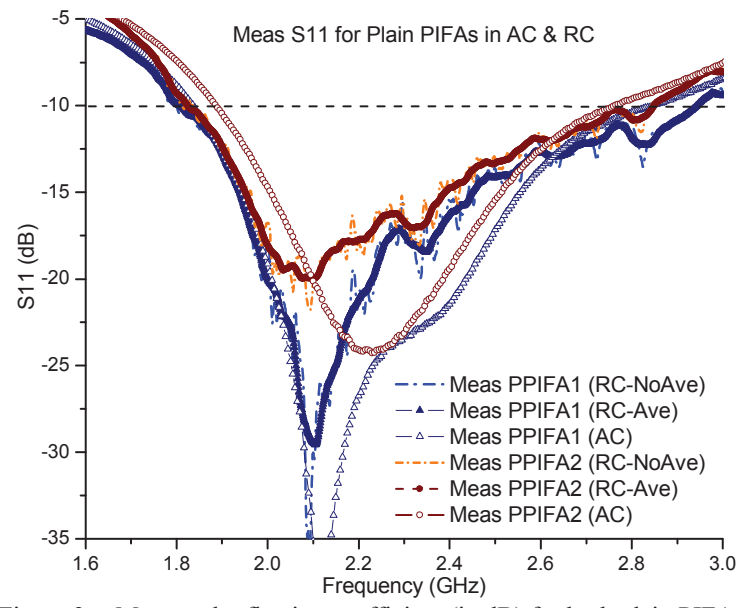

Figure 3. Measured reflection coefficient (in $\mathrm{dB}$ ) for both plain PIFA prototypes

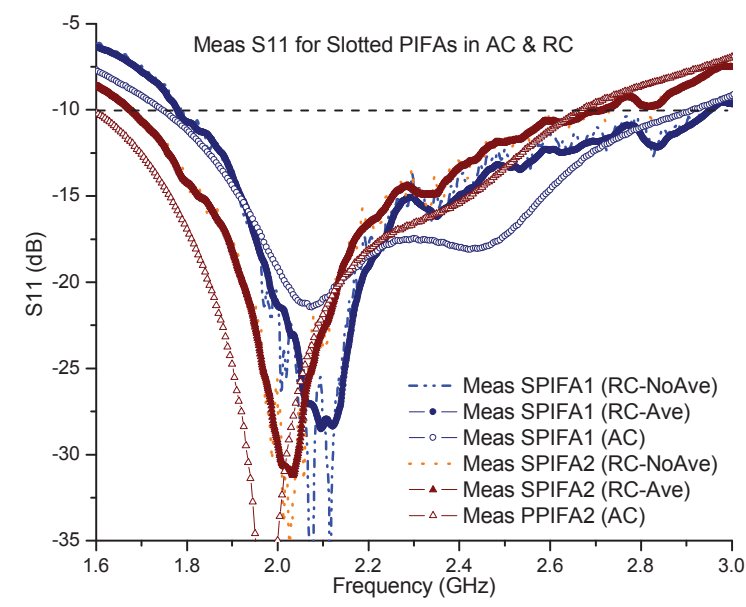

Figure 4. Measured reflection coefficient (in dB) for both slotted PIFA prototypes

TABLE I. Summary of Simulated AND MEASURed PIFA PERFORMANCE

\begin{tabular}{|l|c|c|c|c|}
\hline & PPIFA1 & PPIFA2 & SPIFA1 & SPIFA2 \\
\hline $\begin{array}{l}\text { Sim } \\
(\mathrm{MHz})\end{array}$ & \multicolumn{2}{|c|}{912} & \multicolumn{2}{|c|}{940} \\
\hline $\begin{array}{l}\text { Meas } A C B W \\
(\mathrm{MHz})\end{array}$ & 970 & 1010 & 1160 & 1080 \\
\hline $\begin{array}{l}\text { Meas } R C B W \\
(\mathrm{MHz})\end{array}$ & 1024 & 1134 & 1173 & 1042 \\
\hline Sim $e_{\text {Rad }}(\mathrm{dB})$ & \multicolumn{2}{|c|}{-0.088} & \multicolumn{2}{|c|}{-0.085} \\
\hline Sim $e_{\mathrm{Tot}}(\mathrm{dB})$ & \multicolumn{2}{|c|}{-0.317} & \multicolumn{2}{|c|}{-0.319} \\
\hline Meas $e_{\text {Rad }}(\mathrm{dB})$ & -0.668 & -1.241 & -0.804 & -2.176 \\
\hline Meas $e_{\text {Tot }}(\mathrm{dB})$ & -0.845 & -1.443 & -0.997 & -2.366 \\
\hline
\end{tabular}

average power transfer functions, $(1 / N) \sum_{n=1}^{N}\left|S_{21}\right|^{2}$, of the two measurements is the ratio between the efficiencies of the two antennas. Using both the absorption losses $\left(e_{\mathrm{Rad}}\right)$ and mismatch factor $\left(1-\left|S_{11}\right|^{2}\right)$, the total radiated efficiency $\left(e_{\mathrm{Tot}}\right)$ of the antenna is then obtained through:

$$
e_{T o t}=e_{\text {Rad }}\left(1-\left|S_{11}\right|^{2}\right)
$$

\section{RESUlt AND DiscUSSION}

An accurate value of the reflection coefficient $\left(S_{11}\right)$ is critical to ensure good accuracy. Thus a comparison of $S_{11}$ is carried out. A reflection coefficient measurement is first carried out in an anechoic chamber $(A C)$ available at KU Leuven before it is transported to Chalmers for measurement in the Bluetest $R C$. The $A C$ measurement is carried out using 401 points from 1.5 $\mathrm{GHz}$ to $3.5 \mathrm{GHz}$, which produces a $S_{11}$ reading every $5 \mathrm{MHz}$. In Chalmers, the measurement is carried out within a similar range, but using a $1 \mathrm{MHz}$ frequency resolution. This is labeled as $R C$-NoAve. These $R C$ readings are then smoothened out with a $5 \mathrm{MHz}$ frequency stirring to produce the same resolution as in the $A C$ for comparison, as can be seen in Figs. 3 and 4, being labeled as $R C$-Ave. It can be seen that the measurements at both locations, using both $A C$ and $R C$, are agreeing well with each other, especially above $-10 \mathrm{~dB}$. Below that, it is more difficult to measure $S_{11}$ accurately. A slight $S_{11}$ degradation/shift is seen for the slotted PIFAs due to the presence of the slot, which is not secured onto the fleece substrate. A slight change in performance originating from the transportation between $A C$ and $R C$ is also not ruled out.

Generally, bandwidth $(B W)$ in this work is defined at the $10 \mathrm{~dB}$ borders of the antennas' $S_{11}$. Measurements in both $A C$ and $R C$ are agreeing well, showing more than $1 \mathrm{GHz}$ of $B W$. Differences of less than $50 \mathrm{MHz}$ are seen between both measurements. This is reasonable; considering that the flexible antenna is only supported by an SMA connector and the RF cable at the bottom of the PIFA during measurements. This is done to avoid electromagnetic coupling to nearby objects in both rooms. As a result, rotator movements in $R C$ during measurement have possibly altered the physical conditions of the flexible antenna, resulting in the discrepancy. On the other hand, simulations produced about $100 \mathrm{MHz}$ less bandwidth for both slotted and plain structures. This is due to the modeling assumption that there is almost zero dielectric loss in the fleece substrate.

Efficiency measurements performed in the Bluetest $R C$ of all four antenna prototypes are shown in Figs. 5 and 6, while $e_{\text {Rad }}$ and $e_{\text {Tot }}$ are listed in Table I. It can be clearly seen from the measurements that both plain and slotted prototypes with a larger fleece, and thus total antenna size, are agreeing better with the simulations. Simulated and measured $e_{\text {Tot }}$ differ by about $0.5 \mathrm{~dB}$ for PPIFA1 and about $1 \mathrm{~dB}$ for SPIFA1. On the other hand, a fair agreement is seen for prototype 2. More than $1 \mathrm{~dB}$ and $2 \mathrm{~dB}$ of measurement-simulation difference are seen for PPIFA2 and SSPIFA2, respectively. This indicates that additional substrate area has significantly increased the radiation resistance $\left(R_{\mathrm{r}}\right)$, and in turn, increased radiated power 


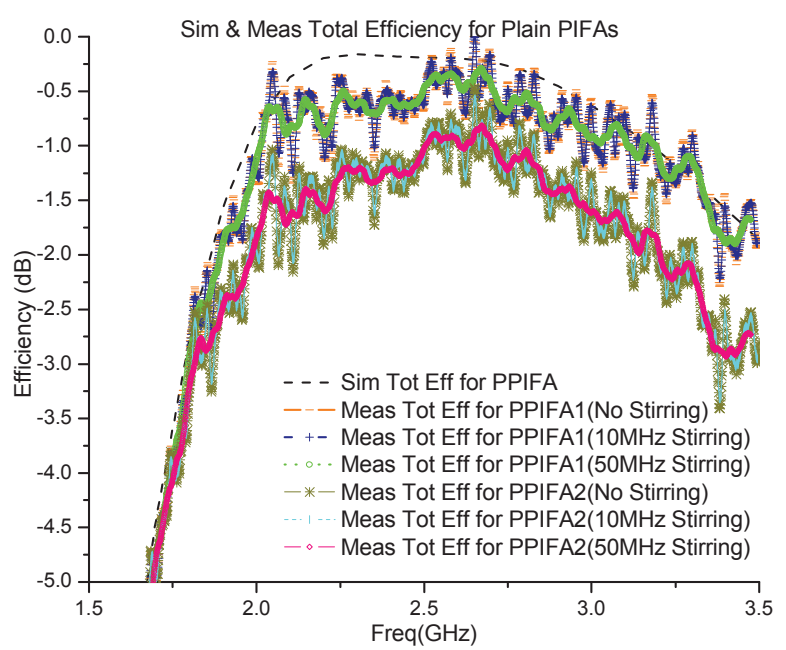

Figure 5. Simulated and measured total efficiency for plain PIFAs

$P_{\mathrm{r}}$. This is assuming a fixed ohmic resistance $\left(R_{\mathrm{Ohm}}\right)$. These quantities are related by:

$$
e_{\text {Rad }}=\frac{R_{r}}{R_{r}+R_{\text {Ohm }}}
$$

Initially, it was thought that the textile and epoxy's ageing would affect the antenna performance over a specified period of time. This is due to the textile, which is plated using layer of pure copper, which could suffer from oxidation, in this way reducing its conductivity. Moreover, the conductive epoxy used for the textile-SMA interconnection might have also degraded mechanically, especially under bending conditions. However, from this investigation, it can be observed that at least these antenna prototypes, fabricated a month apart, did not show any significant degradation. Differences are largely due to fabrication inaccuracies and dielectric substrate's size instead.

Frequency stirring with proper $B W$ can improve measurement accuracy, yet a too large frequency stirring $B W$ will degrade frequency resolution. We examine the frequency stirring effect on the efficiency in Fig. 5 and Fig. 6. Frequency stirring tends to result in a smoother curve, reducing uncertainty due to statistical variations with frequency. For the present antennas, the actual frequency variation of the efficiency is so slow (see theoretical curve) that we can frequency stir $50 \mathrm{MHz}$ or even more.

\section{CONCLUSION}

A set of four planar inverted-F antennas (PIFAs) using conductive textile has been successfully designed, fabricated and tested. Characterization of efficiencies in a reverberation chamber has also been carried out, indicating values larger than $-3 \mathrm{~dB}$. Although the two prototypes are affected by fabrication inaccuracies and the substrate sizes are different, measurement results of the prototypes PPIFAI and SPIFAI indicated an excellent agreement with simulations, with a difference of less than $1 \mathrm{~dB}$. Larger efficiency disparity, of about $0.2 \mathrm{~dB}$, is observed for the slotted antennas, due to the existence of the unsecured slot. This also proved that increased practical tolerances must be catered in the fabrication of more complicated textile antenna structures.

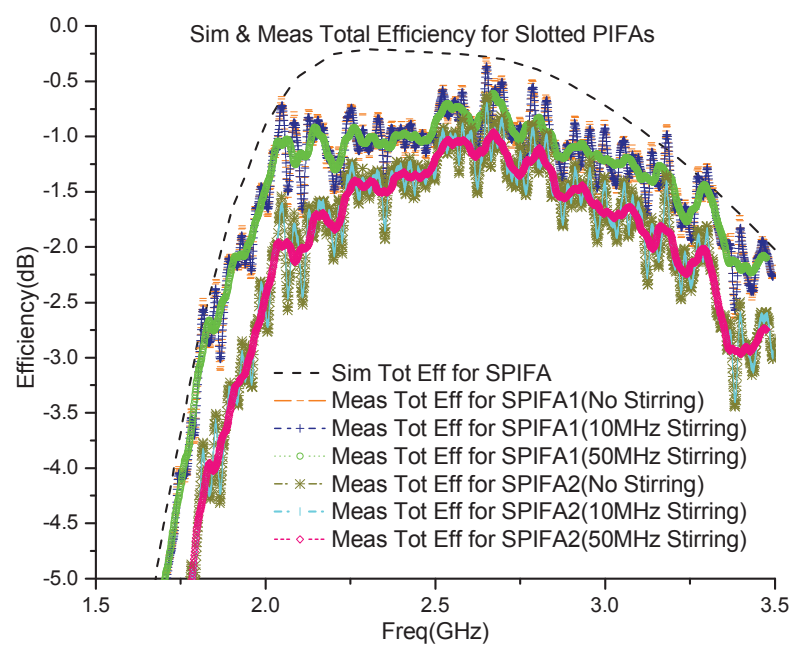

Figure 6. Simulated and measured total efficiency for slotted PIFAs

\section{REFERENCES}

[1] P. J. Soh, G. A. E. Vandenbosch, V. Volskiy, and H. M. R. Nurul, "Characterization of a Simple Broadband Textile Planar Inverted-F Antenna (PIFA) for on Body Communications," in International Conference on Applied Electromagnetics and Communications 2010, 2010.

[2] P. S. Kildal and K. Rosengren, "Correlation and capacity of MIMO systems and mutual coupling, radiation efficiency, and diversity gain of their antennas: simulations and measurements in a reverberation chamber," Communications Magazine, IEEE, vol. 42, pp. 104-112, 2004.

[3] N. Serafimov, P. S. Kildal, and T. Bolin, "Comparison between radiation efficiencies of phone antennas and radiated power of mobile phones measured in anechoic chambers and reverberation chamber," in Antennas and Propagation Society International Symposium, 2002. IEEE, 2002, pp. 478-481 vol.2.

[4] G. A. Conway, W. G. Scanlon, C. Orlenius, and C. Walker, "In Situ Measurement of UHF Wearable Antenna Radiation Efficiency Using a Reverberation Chamber," Antennas and Wireless Propagation Letters, IEEE, vol. 7, pp. 271-274, 2008.

[5] U. Carlberg, P. S. Kildal, and J. Carlsson, "Numerical Study of Position Stirring and Frequency Stirring in a Loaded Reverberation Chamber," Electromagnetic Compatibility, IEEE Transactions on, vol. 51, pp. 1217, 2009.

[6] P.-S. Kildal and C. Carlsson, "Detection of a polarization imbalance in reverberation chambers and how to remove it by polarization stirring when measuring antenna efficiencies," Microwave and Optical Technology Letters, vol. 34, pp. 145-149, 2002.

[7] A. Tronquo, H. Rogier, C. Hertleer, and L. Van Langenhove, "Robust planar textile antenna for wireless body LANs operating in $2.45 \mathrm{GHz}$ ISM band," Electronics Letters, vol. 42, pp. 142-143, 2006.

[8] H. T. Chattha, H. Yi, Z. Xu, and L. Yang, "An Empirical Equation for Predicting the Resonant Frequency of Planar Inverted-F Antennas," Antennas and Wireless Propagation Letters, IEEE, vol. 8, pp. 856-860, 2009. 